\title{
ESTRATÉGIAS DIDÁTICAS IDENTIFICADAS JUNTO A GRADUANDOS DE ENFERMAGEM
}

\author{
Suzel Regina Ribeiro Chavaglia' ${ }^{1}$, Maria Helena Barbosa ${ }^{2}$, Alvaro da Silva Santos ${ }^{3}$, \\ Rafaela Dagma Duarte ${ }^{4}$, Divanice Contim ${ }^{5}$, Rosali Isabel Barduchi Ohl ${ }^{6}$
}

\begin{abstract}
RESUMO: Objetivos: identificar as estratégias, os recursos didáticos e as ferramentas digitais utilizados no ensino de graduação em Enfermagem na opinião dos estudantes. Método: Estudo descritivo, transversal, realizado no período de maio a agosto de 2016, com 149 graduandos de Enfermagem de uma universidade pública de Minas Gerais. Os dados foram analisados de forma descritiva simples pelo Statistical Package for the Social Sciences (SPSS), versão 20.0. Resultados: As estratégias de ensino predominantes foram: as aulas expositivo-dialogadas, 147 (94,6\%) e aulas expositivas 131 (87,9\%). Os recursos de ensino foram: Data Show 148 (99,3\%); leitura de artigos 146 (98\%) e projetor de slides 142 (95,3\%). As ferramentas digitais predominantes foram: a Apresentação Digital PowerPoint, 147 (98,7\%); o e-mail, $145(97,4 \%)$ e a ferramenta de pesquisa Google, com 144 (96,7\%). Conclusão: Os resultados demonstram a necessidade de maior diversificação e utilização de estratégias diversas que empreguem as Tecnologias de Informação e Comunicação no ensino de Enfermagem.
\end{abstract}

DESCRITORES: Tecnologia da informação; Meios de comunicação; Ensino; Educação em enfermagem; Enfermagem.

\section{DIDACTIC STRATEGIES IDENTIFIED BY NURSING STUDENTS}

\begin{abstract}
Objectives: To identify the strategies, didactic resources, and digital tools used in undergraduate nursing teaching, based on the students' opinions. Method: A descriptive, cross-sectional study carried out between May and August 2016, with 149 nursing undergraduates from a public university in the state of Minas Gerais. Data were analyzed in a simple descriptive way by the Statistical Package for the Social Sciences (SPSS), version 20.0. Results: The predominant teaching strategies were: 147 expository-dialogue classes (94.6\%); and 131 expository classes (87.9\%). The teaching resources were: 148 Data Show, 148 (99.3\%); 146 article reading (98\%); and 142 slide projections (95.3\%). The predominant digital tools were: Digital PowerPoint ${ }^{\mathrm{TM}}$ presentation, 147 (98.7\%); e-mail, 145 (97.4\%); and Google search engine, 144 (96.7\%). Conclusion: The results demonstrate the need for greater diversification and use of several strategies that employ information and communication technologies in nursing education. DESCRIPTORS: Information Technology; Means of communication; Teaching; Nursing Education; Nursing.
\end{abstract}

\section{ESTRATEGIAS DIDÁCTICAS IDENTIFICADAS CON ESTUDIANTES AVANZADOS DE ENFERMERÍA}

RESUMEN: Objetivos: Identificar estrategias, recursos didácticos y herramientas digitales utilizadas en enseñanza de grado en Enfermería, según los estudiantes. Método: Estudio descriptivo, transversal, realizado de mayo a agosto de 2016, con 149 estudiantes avanzados de Enfermería de una universidad pública de Minas Gerais. Datos analizados en forma descriptiva simple mediante Statistical Package for the Social Sciences (SPSS), versión 20.0. Resultados: Las estrategias de enseñanza predominantes fueron: clases expositivo-dialogadas, 147 (94,6\%) y clases expositivas, 131 $(87,9 \%)$. Los recursos de enseñanza fueron: Data Show, 148 (99,3\%); lectura de artículos, 146 (98\%) y proyector de diapositivas, 142 (95,3\%). Las herramientas digitales predominantes fueron: Presentación Digital Power Point, 147 (98,7\%); e-mail, 145 (97,4\%) y el motor de búsqueda Google, $144(94,7 \%)$. Conclusión: Los resultados demostraron necesidad de mayor diversificación y utilización de estrategias diferentes que utilicen las Tecnologías de Información y Comunicación en la enseñanza de Enfermería.

DESCRIPTORES: Tecnología de la Información; Medios de Comunicación; Enseñanza; Educación en Enfermería; Enfermería.

${ }^{1}$ Enfermeira. Doutora em Enfermagem. Docente do Departamento de Enfermagem na Assistência Hospitalar da Universidade Federal do Triângulo Mineiro. Uberaba, MG, Brasil.

${ }^{2}$ Enfermeira. Doutora em Enfermagem na Saúde do Adulto. Docente do Departamento de Enfermagem na Assistência Hospitalar da Universidade Federal do Triângulo Mineiro. Uberaba, MG, Brasil.

${ }^{3}$ Enfermeiro. Doutor em Ciências Sociais. Docente do Departamento de Enfermagem em Educação em Saúde da Universidade Federal do Triângulo Mineiro. Uberaba, MG, Brasil.

${ }^{4}$ Enfermeira. Universidade Federal do Triângulo Mineiro. Uberaba, MG, Brasil.

${ }^{5}$ Enfermeira. Doutora em Enfermagem. Docente do Departamento de Enfermagem na Assistência Hospitalar da Universidade Federal do Triângulo Mineiro. Uberaba, MG, Brasil.

${ }^{6}$ Enfermeira. Doutora em Enfermagem. Docente do Departamento de Enfermagem Clínica e Cirúrgica, Escola Paulista de Enfermagem da Universidade Federal de São Paulo. São Paulo, SP, Brasil. 


\section{INTRODUÇÃO}

O processo ensino-aprendizagem na graduação em Enfermagem tem sido fonte de questionamentos motivadores à investigação das estratégias, recursos e Tecnologias da Informação e Comunicação (TICs) utilizados no processo de formação do enfermeiro.

Os meios que o professor utiliza para facilitar o processo de ensino-aprendizagem definem-se como conceito de estratégia de ensino. Nesta definição, incluem-se a organização do espaço de aula, materiais necessários, recursos audiovisuais, visitas técnicas, estudos de casos, discussões em grupos, o uso da Internet e de programas educacionais para computadores, dentre outras inúmeras opções ${ }^{(1)}$.

Estudos relacionados à educação em Enfermagem evidenciam a necessidade de constante aprimoramento do docente em relação a essa temática, bem como a utilização de novas estratégias, recursos e tecnologias de ensino necessários à prática docente ${ }^{(2-3)}$.

As Diretrizes Curriculares Nacionais dos Cursos de Graduação em Enfermagem, ${ }^{(4)}$ aprovadas pelo Conselho Nacional de Educação, destacam a importância do desenvolvimento de estratégias de aproximação dos graduandos, por meio das tecnologias da informação e comunicação, por serem consideradas de extrema importância para a formação do enfermeiro com perfil crítico e reflexivo.

O termo Tecnologias da Informação e Comunicação refere-se à conjunção da tecnologia computacional ou informática com a tecnologia das telecomunicações. Esta definição engloba o uso do rádio, televisão, telefonia (fixa e móvel), computadores e da internet, tendo na Internet e, mais particularmente, no World Wide Web (www), sua mais forte expressão(5).

Grande parte dos professores considera dois grandes obstáculos para o uso das TIC nas práticas de ensino: a falta de infraestrutura e a falta de formação, capacitação específica na área e motivação para a mudança de concepções e práticas de ensino ${ }^{(6)}$.

O desenvolvimento tecnológico e o fácil acesso à informação atualmente têm permitido o contato do estudante com a realidade e o conhecimento além das fronteiras tradicionais da escola e família, visto que esses grupos sociais não constituem os únicos responsáveis pela administração da informação.

As fontes de informação são diversificadas e as instituições formadoras têm o dever de estimular novas estratégias de ensino. Nesse sentido, os educadores necessitam de capacitação, principalmente quando esse ensino possuir modalidade a distância, e devem assumir o papel de orientadores e mediadores responsáveis para indicar, ao estudante, quais parâmetros e critérios de busca para a construção de seu conhecimento diante das diversas fontes de informação existentes ${ }^{(7)}$.

Existe uma diversidade de estratégias, recursos didáticos e ferramentas tecnológicas que podem influenciar positivamente no processo de ensino-aprendizagem. Tais estratégias, recursos didáticos e ferramentas motivam e facilitam o desenvolvimento dos processos mentais como observar, comparar, analisar e sintetizar, propiciar experiências, além de promover a aproximação dos alunos ${ }^{(1,8-10)}$.

Nesse sentido, o uso de ferramentas digitais, como o Ambiente Virtual de Aprendizagem (AVA), softwares como Microsoft Office - Word, PowerPoint, Excel e redes sociais, surge com mais frequênciaatualmente no processo de ensino em geral. Hoje, há mais acesso dos estudantes à internet, fato que propicia o desenvolvimentodo seu aprendizado em outros cenários além da sala de aula, no seu domicílio, em outros ambientes na escola, lugares que tenham computador, tablet ou smartphone com conexão à internet ${ }^{(10)}$.

A Enfermagem é considerada pioneira na utilização de tecnologias educacionais, porém, ainda enfrenta grandes desafios, como o preparo dos profissionais para a prática de saúde em ambientes informatizados. Tornam-se imperativas, aos educadores dessa área, a compreensão e a utilização dos recursos tecnológicos para a prática docente ${ }^{(11)}$.

A adoção das TIC na Enfermagem procura acompanhar o desenvolvimento tecnológico no ensino na graduação, pós-graduação e educação em saúde ${ }^{(12)}$. Assim, o objetivo deste estudo foi identificar as estratégias, os recursos didáticos e as ferramentas digitais utilizados no ensino de graduação em Enfermagem na opinião dos estudantes. 


\section{MÉTODO}

Este é um estudo transversal realizado com os estudantes do Curso de Graduação em Enfermagem de uma universidade pública de Minas Gerais, Brasil. Caracterizou-se o perfil sociodemográfico dos estudantes de graduação em Enfermagem e foram identificados as estratégias de ensino, os recursos didáticos e as ferramentas digitais utilizados de acordo com a opinião dos estudantes.

Participaram da pesquisa os estudantes que atenderam ao seguinte critério de inclusão: estarem regularmente matriculados do $2^{\circ}$ ao $8^{\circ}$ períodos do curso. Entende-se que os alunos do primeiro período vivenciaram poucas disciplinas e estratégias de ensino. Portanto, estes foram excluídos do estudo, assim como os alunos do $9^{\circ}$ e $10^{\circ}$ períodos, por estarem em atividades práticas somente no estágio curricular supervisionado. Outras perdas correspondem àqueles estudantes que não foram encontrados após três tentativas de abordagem dos pesquisadores.

O instrumento de coleta de dados foi estruturado em duas partes. A partel, com dados sociodemográficos contendo seis questões, com uma das questões interrogando como o aluno classifica seu conhecimento acerca das Tecnologias da Informação e Comunicação, e as opções de resposta eram: Excelente, Bom, Suficiente, Pouco e Nenhum. A parte II contou com quatro questões fechadas com diversas alternativas acerca das estratégias de ensino, recursos didáticos e as ferramentas digitais e três possibilidades de resposta: Sim, Não e Não conheço. Anexo à parte II, foi disponibilizado um tutorial com as definições dos itens investigados.

Realizou-se a coleta de dados junto aos alunos de cada período, em horário e local previamente agendados, entre os meses de maio a agosto de 2016.

Os dados foram registrados em planilha eletrônica do programa Microsoft Exce/ß e analisados pelo programa Statistical Package for the Social Sciences (SPSS), versão 20.0, realizando-se análise estatística descritiva simples.

O estudo foi realizado respeitando-se os princípios éticos e aprovado pelo Comitê de Ética e Pesquisa da Universidade Federal do Triângulo Mineiro sob o n.․․ 2746/13.

\section{RESUlTADOS}

De um total de 274 alunos regularmente matriculados, participaram da pesquisa 149 estudantes. Os alunos não participantes (125) se encontravam dentro do critério de exclusão.

O período com maior número de alunos participantes da pesquisa foi o $6^{\circ}$ período, com 29 (19,5\%) estudantes, e o menor foi o 5 o período, com 14 (9,4\%) estudantes. Predominou o sexo feminino, com 132 $(88,6 \%)$ participantes, e a faixa etária entre 19 e 24 anos, com $121(81,2 \%)$ sujeitos. O conhecimento sobre as Tecnologias da Informação e Comunicação - TIC foi autorreferido pelos participantes a partir das respostas: Excelente, Bom, Suficiente, Pouco e Nenhum. Já a maioria das avaliaçãoes foi: Bom, com $65(43,4 \%)$ respostas, seguido de Suficiente, com 41 (27,5\%).

As estratégias didáticas mais frequentes foram a aula expositivo-dialogada, 147 (94,6\%); aula expositiva, 131 (87,9\%), e exposição e visitas, com $130(87,3 \%)$ respostas. As estratégias menos indicadas ou desconhecidas foram "mapa conceitual" e "dramatização Role Play", com a mesma frequência e percentual, 51 (34,2\%),e jogos educativos, com 55 (36,9\%).Em relação ao ambiente de aprendizagem, houve a predominância do ambiente presencial (Tabela 1). Vale ressaltar que somente a estratégia "aula expositivo-dialogada" obteve resposta de quase a totalidade dos participantes da pesquisa. 
Tabela 1 - Estratégias e recursos de ensino e o ambiente em que foram desenvolvidos, conforme os graduandos de Enfermagem. Uberaba, MG, 2016

\begin{tabular}{ccccc}
\hline Estratégias e Recursos de Ensino & $\begin{array}{c}\text { Ambiente } \\
\text { Presencial } \\
(\mathbf{n})(\%)\end{array}$ & $\begin{array}{c}\text { Ambiente Virtual e } \\
\text { Presencial } \\
(\mathbf{n})(\%)\end{array}$ & $\begin{array}{c}\text { Ambiente } \\
\text { Virtual } \\
(\mathbf{n})(\%)\end{array}$ & $\begin{array}{c}\text { Sem } \\
\text { Resposta }\end{array}$ \\
\hline Aula expositivo-dialogada & $141(94,6)$ & $1(0,7)$ & $7(4,7)$ & $0(0)$ \\
Aula expositiva & $131(87,9)$ & $5(3,3)$ & $4(2,7)$ & $9(6,1)$ \\
Exposição e visitas & $130(87,3)$ & $2(1,3)$ & $2(1,3)$ & $15(10,1)$ \\
Simulação em laboratório & $127(85,3)$ & $0(0)$ & $2(1,3)$ & $20(13,4)$ \\
Seminário & $122(81,9)$ & $12(8,1)$ & $7(4,7)$ & $8(5,3)$ \\
Solução de problemas & $120(80,6)$ & $10(6,7)$ & $2(1,3)$ & $17(11,4)$ \\
Estudo de caso & $117(78,5)$ & $9(6)$ & $4(2,7)$ & $19(12,8)$ \\
Estudo de texto & $117(78,6)$ & $13(8,7)$ & $8(5,3)$ & $11(7,4)$ \\
Estudo dirigido & $114(76,5)$ & $7(4,7)$ & $9(6)$ & $19(12,8)$ \\
Ensino clínico & $112(75,2)$ & $0(0)$ & $4(2,7)$ & $33(22,1)$ \\
Outros & $112(75,1)$ & $8(5,41)$ & $7(4,7)$ & $149(100)$ \\
Portfólio & $108(72,5)$ & $5(3,3)$ & $17(11,4)$ & $19(12,8)$ \\
Grupo de verbalização e & $96(64,4)$ & $1(0,7)$ & $6(4,1)$ & $46(30,8)$ \\
observação & $95(63,7)$ & $15(10,1)$ & $18(12,1)$ & $21(14,1)$ \\
Ensino com pesquisa & $92(61,7)$ & $4(2,7)$ & $4(2,7)$ & $49(32,9)$ \\
Fórum de Discussão/debates & $88(59,2)$ & $5(3,3)$ & $2(1,3)$ & $54(36,2)$ \\
Estudo do meio & $81(54,4)$ & $22(14,7)$ & $10(6,7)$ & $36(24,2)$ \\
Orientação & $68(45,7)$ & $10(6,7)$ & $14(9,4)$ & $57(38,2)$ \\
Ensino individualizado & $57(38,2)$ & $0(0)$ & $2(1,3)$ & $90(60,5)$ \\
Júri simulado & $55(36,9)$ & $2(1,3)$ & $3(2)$ & $89(59,8)$ \\
Jogos educativos & $51(34,2)$ & $0(0)$ & $8(5,3)$ & $90(60,5)$ \\
Mapa conceitual & $51(34,2)$ & $2(1,3)$ & $9(6)$ & $87(58,5)$ \\
\hline Dramatização - Role Play & & &
\end{tabular}

Com relação aos recursos didáticos utilizados, houve a predominância do uso de Data Show, com 148 (99,3\%) indicações, seguido da leitura de artigos,146 (98\%), e uso de projetor de slides e a realização de exercícios, com a mesma frequência e percentual: 142 (95,3\%). O uso de smartboard foi o que obteve a menor indicação, nove $(6,0 \%)$, conforme a tabela 2 .

Tabela 2 - Recursos didáticos utilizados de acordo com os graduandos de Enfermagem. Uberaba, MG, 2016. (coninua)

\begin{tabular}{|c|c|c|c|}
\hline & $\begin{array}{c}\text { Sim } \\
\text { (n) }(\%)\end{array}$ & $\begin{array}{c}\text { Não } \\
\text { (n) (\%) }\end{array}$ & $\begin{array}{l}\text { Não conheço } \\
\text { (n) }(\%)\end{array}$ \\
\hline Data show & $148(99,3)$ & $1(0,7)$ & $0(0)$ \\
\hline Artigos & $146(98)$ & $3(2)$ & $0(0)$ \\
\hline Exercícios para fixação de conteúdo & $142(95,3)$ & $7(4,7)$ & $0(0)$ \\
\hline Projetor de Slides & $142(95,3)$ & $7(4,7)$ & $0(0)$ \\
\hline Filmes/Vídeos & $141(94,6)$ & $8(5,4)$ & $0(0)$ \\
\hline Leituras complementares & $141(94,6)$ & $8(5,4)$ & $0(0)$ \\
\hline Quadro negro/branco & $136(91,3)$ & $13(8,7)$ & $0(0)$ \\
\hline Livro texto & $131(87,9)$ & $18(12)$ & $0(0)$ \\
\hline
\end{tabular}




$\begin{array}{cccc}\text { Retroprojetor } & 115(77,2) & 34(22,8) & 0(0) \\ \text { Laboratório de informática } & 109(73,2) & 40(26,8) & 0(0) \\ \text { Apostilas } & 70(47) & 79(53) & 0(0) \\ \text { Smartboard } & 9(6) & 138(92,7) & 2(1,3)\end{array}$

As ferramentas digitais mais frequentes foram a apresentação digital, por meio do software Microsoft Office - Power Point,147(98,7\%); E-mail, 145 (97,4\%), e ferramenta de pesquisa Google, com 144 $(96,7 \%)$ indicações. Já as ferramentas digitais menos frequentes foram a videoconferência e fórum online, ambas com o mesmo percentual, 23 (15,4\%), como exposto na Tabela 3.

Tabela 3 - Uso de ferramentas digitais segundo os graduandos em Enfermagem. Uberaba, MG, 2016

\begin{tabular}{lccc}
\hline & $\begin{array}{c}\text { Sim } \\
(\mathbf{n})(\%)\end{array}$ & $\begin{array}{c}\text { Não } \\
(\mathbf{n})(\%)\end{array}$ & $\begin{array}{c}\text { Não Conheço } \\
\text { (n) }(\%)\end{array}$ \\
\hline Apresentação Digital (PowerPoint) & $147(98,7)$ & $1(0,7)$ & $1(0,7)$ \\
E-mail & $145(97,4)$ & $2(1,3)$ & $2(1,3)$ \\
Google & $144(96,7)$ & $2(1,3)$ & $3(2)$ \\
YouTube & $130(87,3)$ & $17(11,4)$ & $2(1,3)$ \\
Redes Sociais & $123(82,6)$ & $22(14,7)$ & $4(2,7)$ \\
Slide Share & $84(56,4)$ & $31(20,8)$ & $34(22,8)$ \\
Scribd & $75(50,3)$ & $43(28,9)$ & $31(20,8)$ \\
Moviemaker & $71(47,6)$ & $56(37,6)$ & $22(14,8)$ \\
Ambiente Moodle & $47(31,5)$ & $73(49)$ & $29(19,5)$ \\
E-book & $33(22,1)$ & $91(61,1)$ & $25(16,8)$ \\
Blog & $28(18,8)$ & $107(71,8)$ & $14(9,4)$ \\
Videoconferência & $23(15,4)$ & $109(73,2)$ & $17(11,4)$ \\
Fórum on-line & $23(15,4)$ & $100(67,1)$ & $26(17,5)$ \\
\hline
\end{tabular}

\section{DISCUSSÃO}

Na atualidade, a comunicação, bem como o acesso à informação por meio da rede de informática, tem sido facilitada e disseminada entre a população, principalmente por meio de ferramentas digitais como o uso da internet, e-mails, videoconferência, aplicativos sociais, entre outros. Esse fato determinou um novo perfil entre os estudantes, hoje considerados como "nativos digitais", ou seja, os nascidos a partir da década de 90, em decorrência do acesso à informação,por meio da $W e b^{(12)}$.

Esse novo perfil de estudantes demanda, dos órgãos formadores e de seus docentes, a aplicação de novas estratégias de ensino, recursos e metodologias, associadas às TIC e aos novos ambientes de aprendizagem, o que se configura em desafio para as instituições de ensino superior.

Estudo realizado na região Sul do Brasil destaca que, a partir do advento das Diretrizes Curriculares Nacionais (DCNs) para a área da saúde, um novo modelo educacional tem sido requerido para superar o vigente até então, tradicional e tecnicista, baseado somente na transmissão de informações e na relação unilateral entre professor-aluno ${ }^{(12)}$. Os dados dessa pesquisa demonstram que os estudantes reconhecem a maioria das estratégias e recursos no ambiente presencial para a maioria das estratégias de ensino, recursos de ensino e ferramentas digitais, cuja pontuação vai da estratégia aula expositivodialogada, 141 (94,6\%), até Dramatização - Role Play, 51 (34,2\%).

Ressalta-se o não reconhecimento pelos estudantes de estratégias de ensino como os jogos educativos, role play, júri simulado e mapa conceitual. Esses dados evidenciam que as metodologias tradicionais de ensino ainda são preponderantes no processo de formação do enfermeiro na instituição estudada. 
Acredita-se que mudanças no processo ensino-aprendizagem da Enfermagem são necessárias, em especial na instituição investigada, e que estratégias sejam selecionadas a partir de metodologias ativas de ensino e da apropriação de diferentes recursos das TIC. Essas estratégias permitem a facilitação da aprendizagem, intensificam o desenvolvimento de capacidades intelectuais e sensitivas dos estudantes, motivando e estimulando sua criatividade e permitindo o alcance de autonomia e a aquisição de conhecimentos, habilidades e atitudes ${ }^{(13)}$.

Destaca-se a necessidade de uma postura assertiva do docente diante dessas novas tecnologias, sobretudo como mediador do processo de aprendizagem, bem como dos dirigentes das instituições de ensino para que haja maiores investimentos na infraestrutura dessas instituições ${ }^{(14)}$.

Estudos apontam para a subutilização das TIC no ensino de cursos de graduação na área da Saúde e da Enfermagem, o que vem ao encontro dos dados obtidos nesta pesquisa, que evidencia o uso das TIC em seminários e aulas expositivo-dialogadas ${ }^{(14-15)}$.

O acesso digital nas instituições de ensino é condição fundamental para que os estudantes de Enfermagem se tornem profissionais capacitados e habilitados às exigências do mercado de trabalho atual. São necessários o aprimoramento e a capacitação do professor em relação a esses recursos tecnológicos para a adoção de novas estratégias de ensino na Enfermagem, bem como os docentes devem estar abertos à mudança, no sentido de renovar continuamente seus planos de ensino, permitindo a inserção das $\operatorname{TIC}^{(16-17)}$.

Neste estudo, predominou a indicação do ambiente presencial no processo de ensino-aprendizagem. Os cursos de graduação em Enfermagem vêm utilizando os Ambientes Virtuais de Aprendizagem (AVA) como meio de facilitação do aprendizado, mas não na instituição investigada. Os AVA são considerados ferramenta de suporte ao ensino presencial importante na formação de futuros enfermeiros com competências digitais para aplicar na sua prática assistencial ${ }^{(18)}$.

Vale ressaltar que o Conselho Federal de Enfermagem (COFEN) se posiciona contrariamente aos cursos da área da saúde exclusivamente a distância por considerar a metodologia inadequada à aprendizagem teórico-prática estabelecida por esses cursos, mas não é contrária à EaD e reconhece a importância da utilização de novas tecnologias educacionais para a formação dos profissionais que lidam diretamente com a vida humana ${ }^{(19)}$.

Dentre os recursos didáticos identificados, predominaram Data Show, leitura de artigos, exercícios de fixação de conteúdo e uso de projetor de slides. Sabe-se que o uso do Data show ou de softwares educacionais, planilhas eletrônicas e outros recursos tecnológicos recentes não se configura em reais modificações no processo de ensino-aprendizagem ou inovação nas ações pedagógicas do docente, principalmente se esses recursos forem utilizados para a reproduzir situações de ensino tradicionais ${ }^{(20)}$.

Porém, os dados obtidos neste estudo indicam um distanciamento, por parte dos docentes, na utilização de novos recursos didáticos e modernas ferramentas digitais nas atividades de ensino no curso de Enfermagem, o que leva a inferir que,no curso de Enfermagem da instituição analisada, os docentes não estão familiarizados com os AVA e as novas tecnologias de ensino-aprendizagem.

As ferramentas digitais identificadas nesta pesquisa, relacionadas às TIC, foram as apresentações por meio do software Microsoft Office - Power Point ${ }^{\mathrm{TM}}$, uso do E-mail e do website Google. Esses dados coincidem com estudo que observou o uso de computador por docentes e alunos em sala de aula apenas quando precisam apresentar alguma tarefa com auxílio do Data Show, com slides elaborados e apresentados por meio do Power Point ${ }^{\mathrm{TM}(21)}$.

Outro dado significativo, obtido nesta pesquisa,é a não identificação das ferramentas digitais Videoconferência, Fórum on-line e Blog pelo estudante no desenvolvimento das ações de ensino. Pode-se inferir que eles não reconhecem tais ferramentas pela indisponibilidade de equipamentos que permitam o uso desses recursos digitais ou, ainda, pelo desconhecimento e despreparo do docente na utilização dessas ferramentas em sua prática de ensino.

A videoconferência tem sido cada vez mais utilizada nos âmbitos empresarial, judicial e educacional. Tem, como vantagem, a economia, é muito utilizada nos programas de pós-graduação em Saúde e Enfermagem, por possibilitar a interação sem a necessidade de deslocamentos, propiciando a participação 
de pessoas distantes geograficamente e otimizando o tempo destinado para os estudos. Porém, vale ressaltar que, para a realização de videoconferências, torna-se necessária toda uma infraestrutura, em quesão necessários equipamentos, como computadores com acesso à internet, rede de banda larga, microfones e câmeras instalados, que propiciem a captura e a reprodução de áudio e vídeo e ambientes virtuais de aprendizagem e pessoal capacitado que possa operar adequadamente esses equipamentos ${ }^{(22)}$.

Estudo que avaliou a educação permanente da equipe de Enfermagem em Unidades Básicas de Saúde, na modalidade a distância, evidenciou que a videoconferência e a teleconsultoria apresentaram impacto positivo na qualificação do pessoal. Esses resultados reforçam a questão da capacitação docente para o uso das TIC na instituição investigada ${ }^{(23)}$.

Os Fóruns on-line e os Blogs são ferramentas digitais que apoiam a abordagem de temas de estudopelo professor no Ambiente Virtual de Aprendizagem. Para tanto, é necessário o conhecimento de todas as potencialidades e aplicações dos recursos virtuais para o incremento do processo de ensinoaprendizagem. A efetiva interação entre os sujeitos no ambiente on-line possibilita a construção do conhecimento pelo estudante mediada pelo professor ${ }^{(24)}$.

É fundamental que o ensino em saúde incorpore os avanços pedagógicos das metodologias ativas de ensino-aprendizagem estabelecendo novas diretrizes para a integração entre teoria e prática e rompendo com a tradicional dicotomia entre ensino básico e formação clínica. As universidades devem adotar metodologias que favoreçam o desenvolvimento do espírito crítico, da capacidade de reflexão e a participação ativa dos estudantes na construção do conhecimento ${ }^{(25)}$.

A utilização de tecnologias incorporadas ao ensino de Enfermagem, como ferramentas da Web 2.0 tais como e-portfólio, web-podcasting, wiki, Special Interest Group (SIG), tele-Enfermagem, a simulação realística, Objective Structured Clinical Evaluation (OSCE), teleimersão e ambientes virtuais, permite a criação de um ambiente inovador, crítico e reflexivo que permite ressignificar o processo de ensinoaprendizagem em Enfermagem ${ }^{(25)}$.

Nesse sentido, outras investigações sobre esta temática devem ser realizadas e sugere-se enfatizar os recursos tecnológicos e metodologias ativas de aprendizagem. Instigar o uso de ferramentas digitais, como blogs, chat, videoaulas, homepages, e-book interativo, webquest, aplicativos e softwares educacionais e jogos digitais, deve ser incentivado a fim de que essas ferramentas possam ser utilizadas no processo de ensino-aprendizagem possibilitando avanços na qualidade do ensino da graduação em Enfermagem ${ }^{(26)}$.

\section{CONCLUSÃO}

Este estudo permitiu identificar as estratégias de ensino, os recursos didáticos e as ferramentas digitais utilizados na graduação em Enfermagem, de uma instituição pública de ensino, a partir da opinião dos estudantes.

Os dados demonstraram a necessidade de maior conhecimento sobre a diversidade de estratégias de ensino, recursos didáticos e uso das ferramentas digitais pelos docentes desse curso a fim de promover maior interação entre professores e estudantes em ambientes presenciais e virtuais de aprendizagem.

Há a necessidade, também, de viabilização de esforços e de investimentos no sentido de prover recursos para a formação permanente dos docentes nessa temática garantindo um melhor preparo docente frente às inovações tecnológicas ofertadas no campo educacional.

Este estudo tem como limitação a impossibilidade de generalizações. No entanto, acredita-se que a realidade aqui retratada também possa ser encontrada no cenário nacional do ensino superior em Enfermagem. Tal fato sugere arealização de outros estudos que comparem várias instituições e, também, outras áreas de formação. 


\section{REFERÊNCIAS}

1. Mazzioni S. As estratégias utilizadas no processo de ensino-aprendizagem: concepções de alunos e professores de ciências contábeis. ReAT. [Internet] 2013;2(1) [acesso em 01 mar 2016]. Disponível: https://periodicos.ufpel.edu.br/ ojs2/index.php/AT/article/view/1426.

2. Grossi LM, Pisa IT, Marin HF. Information and Communication Technology in Nursing Audi. J. Health Inform. [Internet] 2015;7(1):30-4 [acesso em 01 mar 2016]. Disponível: http://www.jhi-sbis.saude.ws/ojs-jhi/index.php/jhi-sbis/ article/view/314/227.

3. Ohl RIB, Cohrs CR, Domenico E, Ferreira P, Bertomeu J. Software CMAP TOOLS ® para construção de mapa conceitual: avaliação pelos estudantes de enfermagem. Anais SBIE. [Internet] 2010;10(1) [acesso em 01 mar 2016]. Disponível: http://www.br-ie.org/pub/index.php/sbie/article/view/1498.

4. Ministério da Educação (BR), Conselho Nacional de Educação. Resolução n.ํㅜ 3, de 07 de novembro de 2001. Institui as Diretrizes Curriculares Nacionais do curso de graduação em enfermagem. Brasília: Ministério da Educação; 2001.

5. dos Santos A. Tecnologias de informação e comunicação: limites e possibilidades no ensino superior. REBES. [Internet] 2015;1(1) [acesso em 01 mar 2016]. Disponível: https://seer.imed.edu.br/index.php/REBES/article/ view/839/630.

6. Albinio R, de Souza CA. Evaluation of the communication and information technology usage in brazilian schools: an exploratory data analysis of the technology for education Brazilian report. E\&G Economia e Gestão. [Internet] 2016;16(43):101-25 [acesso em 01 mar 2016]. Disponível: http://periodicos.pucminas.br/index.php/economiaegestao/ article/view/P.1984-6606.2016v16n43p101/9930.

7. Martins C, Mangan PKV. Institutional Strategies for Continuous Formation for Teachers: a case study in distance learning. Rev. EducaOnline. [Internet] 2015;9(1):32-50 [acesso em 01 mar 2016]. Disponível: http://www.latec.ufrj.br/ revistas/index.php?journal=educaonline\&page=article\&op=view \&path\%5B\%5D=636\&path\%5B\%5D=652.

8. Nogueira MCS. Estratégias de ensinagem aplicadas nas instituições de ensino superior-IES. Rev. Científica da Faculdade de Balsas. [Internet] 2016;6(1) [acesso em 21 jun 2017]. Disponível: http://revista.unibalsas.edu.br/revista/ index.php/unibalsas/article/view/144/87.

9. Silva A. Da aula convencional para a aula invertida - ferramentas digitais para a aula de hoje. Série-Estudos [Internet] 2015;39 [acesso em 21 jun 2017]. Disponível: http://www.serie-estudos.ucdb.br/index.php/serie-estudos/ article/view/840/690.

10. Beserra GL, Ponte BAL, da Silva RP, Beserra EP, de Sousa LB, Gubert FA. Atividade de vida "comunicar" e uso de redes sociais sob a perspectiva de adolescentes. Cogitare Enferm. [Internet] 2016;21(1) [acesso em 01 jun 2017]. Disponível: http://dx.doi.org/10.5380/ce.v21i4.41677.

11. Jensen R, Guedes ES, Leite MMJ. Informatics competencies essential to decision making in nursing management. Rev. esc. enferm. USP. [Internet] 2016;50(1):109-17 [acesso em 01 mar 2016]. Disponível: http://dx.doi.org/10.1590/ S0080-623420160000100015.

12. Jacondino M, Silveira DN, Martins CL, Coimbra VCC. Processo de ensino-aprendizagem do estudante de enfermagem e os estilos de aprendizagem. Journal of Learning Styles. [Internet] 2015;8(15) [acesso em 01 mar 2016]. Disponível: http://learningstyles.uvu.edu/index.php/jls/article/view/222.

13. Vendruscolo C, Trindade LL, Prado ML, Luz JH, Dal Sasso GTM, Erdmann AL. A informática na formação e qualificação dos profissionais de saúde: uma revisão integrativa. Rev. Enferm. UFSM. [Internet] 2013;3(3):539-46 [acesso em 01 mar 2016]. Disponível: http://dx.doi.org/10.5902/217976927465.

14. Andrade EMLR, Nascimento CS. Use of information and communication technologies for nursing students. Rev. Enferm. UFPI. [Internet] 2014;3(1):59-64 [acesso em 01 mar 2016]. Disponível: https://doi.org/10.26694/reufpi. v3i1.1443.

15. Gonçalves LS, Castro TC, Fialek SA. Experiência computacional de enfermeiros brasileiros de um Hospital Universitário. J. Health Inform. [Internet] 2015;7(3):82-87 [acesso em 01 mar 2016]. Disponivel: http://www.jhi-sbis. saude.ws/ojs-jhi/index.php/jhi-sbis/article/view/342. 
16. da Costa CPV, Luz MHBA. Digital learning object for diagnostic reasoning in nursing applied to the integumentary system. Rev. Gaúcha Enferm. [Internet] 2015;36(4):55-62 [acesso em 01 mar 2016]. Disponível: http://dx.doi. org/10.1590/1983-1447.2015.04.54128

17. Holanda VR, Pinheiro AKB, Fernandes AFC, Holanda ER, Souza MA, dos Santos SMJ. Analysis of the national scientific production on the utilization of digital technologies for nurses' education. Rev. Eletr. Enf. [Internet] 2013;15(4):1068-76 [acesso em 01 mar 2016]. Disponível: https://doi.org/10.5216/ree.v15i4.22448.

18. Orosco SS, Puglia MF, Duarte KLG. Hybrid course in nursing: assessment of quality according to the indicators system. Rev. Enferm. UFSM. [Internet] 2014;4(2):265-75 [acesso em 01 mar 2016] Disponível: http://dx.doi. org/10.5902/2179769212903.

19. Conselho Federal de Enfermagem. Seminário discute impacto da formação EaD em Enfermagem. 2016. [acesso em 01 mar 2016]. Disponível: http://www.cofen.gov.br/seminario-discute-impacto-da-formacao-ead-emenfermagem_39343.htm.

20. Mühlbeier ARK, Medina RD, Mozzaquatro PM, de Oliveira LC, Moreira RC. Mobile HQ: o uso de softwares educativos na modalidade M-Learning. Revista Informatica Aplicada. [Internet] 2014;10(1):48-55 [acesso em 01 mar 2016]. Disponível: http://ria.net.br/index.php/ria/article/view/114/129. Doi: 10.5753/cbie.sbie.2012.\%25p.

21. Carabetta Júnior V. Using concept maps as a teaching resource for building and interrelating concepts. Rev. Bras. Educ. Med. [Internet] 2013;37(3):441-47 [acesso em 01 mar 2016]. Disponível: http://dx.doi.org/10.1590/S010055022013000300017.

22. Landeiro MJL, Freire RMA, Martins MM, Martins TV, Peres HHC. Educational technology in care management: technological profile of nurses in portuguese hospitals. Rev. Esc. Enferm. USP. [Internet] 2015;49(Esp2):148-53 [acesso em 01 mar 2016]. Disponível: http://dx.doi.org/10.1590/S0080-623420150000800021.

23. Godoy SCB, Guimarães EMP, Assis DSS. Evaluation of the training of nurses in basic health units through telenfermagem. Esc. Anna. Nery. [Internet] 2014;18(1):148-55 [acesso em 01 mar 2016]. Disponível: http://www.scielo. br/pdf/ean/v18n1/1414-8145-ean-18-01-0148.

24. Ruiz-Moreno L, Leite MTM, Ajzen C. Formação didático-pedagógica em saúde: habilidades cognitivas desenvolvidas pelos pós-graduandos no ambiente virtual de aprendizagem. Ciência \& Educação. [Internet] 2013;19(1):217-29 [acesso em 01 mar 2016]. Disponível: http://dx.doi.org/10.1590/S1516-73132013000100015.

25. Salvador PTCO, Martins CCF, Alves KYA, Pereira MS, Santos VEP, Tourinho FSV. Tecnologia no ensino de enfermagem. Rev Baiana Enf. [Internet] 2015;29(1):33-41 [acesso em 20 fev 2018]. Disponivel: https://portalseer.ufba. br/index.php/enfermagem/article/view/9883.

26. Holanda VR, Pinheiro AKB, Holanda ER, Santos MCL. Ensino e aprendizagem em ambiente virtual: atitude de acadêmicos de enfermagem. [Internet] Rev. Min. Enferm. 2015;19(1):141-147. [acesso em 20 fev 2018] Disponivel: http://www.dx.doi.org/10.5935/1415-2762.20150012. 